\title{
A CASE REPORT OF GIANT GENITAL WARTS
}

\section{Luca Grimaldi, ${ }^{1}$ Roberto Cuomo, ${ }^{1}$ Roberta Bilenchi, ${ }^{2}$ Franco Roviello, ${ }^{3}$ Giuseppe Nisi, ${ }^{1}$ Cesare Brandi, ${ }^{1}$ Carlo D'Aniello ${ }^{1}$}

\author{
${ }^{1}$ Department of Plastic and Reconstructive Surgery, University of Siena; ${ }^{2}$ Department of Dermatology, \\ University of Siena; ${ }^{3}$ Department of Oncological Surgery, University of Siena, Italy
}

\begin{abstract}
Giant genital warts (GGW) represent a rare form of sexually transmitted disease caused by the human papillomavirus, arising more frequently in the vulvar and perianal regions as large exophytic cauliflower-like mass. Estimated rate of recurrence is 60 to $66 \%$, while malignant transformation is possible and it has been reported in 30 to $56 \%$ of cases. A 45 years-old woman was admitted to our Structure of Plastic and Reconstructive Surgery, Siena, Italy with an extensive cauliflower-like masses diffused on vulvar and perianal region. The patient was treated in general anaesthesia, with a wide en bloc excision up to free clinical edges and immediate reconstruction of the vulvar continuity. Buschke Lowenstein tumour or giant genital warts is a sporadic tumour with an elevate local recurrence rate. In some cases, surgery can be very difficult and it must be associated to other strategies. An accurate follow-up is always necessary.
\end{abstract}

Key words: Giant genital warts, acuminatum condyloma, Buschke-Lowenstein tumor.

\section{INTRODUCTION}

Giant genital warts (GGW) represent a rare form of sexually transmitted disease caused by the human papillomavirus (HPV), arising more frequently in the vulvar and perianal regions as large exophytic cauliflower-like mass. HPV serotypes 6 and 11 are the pathogens responsible of this disease. Buschke and Lowenstein described giant genital warts in 1925 [1]. This tumour shows high growth speed and a high recurrence after treatment, so wide wounds with clear margins are often necessary for a complete surgical excision [2]. Buschke-Lowenstein tumour (BLT) shows an incidence of $0.1 \%$, has an invasive growth and high recurrence after treatment. Estimated rate of recurrence is 60 to $66 \%$ [3] while malignant transformation is possible and it has been reported in 30 to $56 \%$ of cases $[4,5]$.

\section{CASE REPORT}

A 45-years-old heterosexual woman was admitted to the Structure of Plastic and Reconstructive Surgery of University of Siena (Italy) in October 2013, complaining of genital discomfort. Three years before, the patient had noticed the evidence of little papillary lesions in the perivulvar region, which became progressively more numerous and voluminous, extending to the perianal region as giant, irregularly ovular masses. A clinical examination revealed extensive cauliflower-like masses diffused on vulvar and perianal region, irregular borders, colour ranging from purple to pink (Figure 1).

There was no propagation into the anal canal or into the vagina. The lesions caused difficulty in sitting and compromised the patient's sexual activity. There wasn't clinical lymphadenopathy; past medical history was negative for other sex transmitted diseases and immunosuppression. The patient was treated in general anaesthesia, with a wide en bloc excision up to free clinical edges and immediate reconstruction of the vulvar continuity. The surgical cutting of the skin was made with a scalpel, while cutting and coagulation of the dermis and subcutaneous tissues was performed with an electric scalpel. The large wounds were closed by suturing the external portion of the labia minora with the medial aspect of the labia majora and it was not necessary to resort to surgical flaps. In order to minimize the risk of faecal contamination of the wound, a stoma was harvested and maintained for one month (Figure 2).

The patient underwent periodical checks to reveal any local recurrence (Figure 3).

Histopathological examination reveals an epidermal hyperplasia with para and hyperkeratosis. A lymphoistiocytic inflammatory infiltrate was present, with spikes produced by papillae rich koilocytes.

Correspondence to:

Roberto Cuomo

Department of General and Specialistic Surgery, Plastic Surgery Unit, "Santa Maria alle Scotte" Hospital, University of Siena Viale Bracci n.1, 53100 Siena, Italy.

Tel./Fax: +39.0577 .585158$

E-mail: robertocuomo@outlook.com 


\section{DISCUSSION}

In 1925 a lesion locally invasive, with rapid growing was first described by Abraham Buschke and Ludwig Lowenstein as "carcinoma-like condylomata acuminate" now Buschke-Lowenstein tumour (BLT). BLT is a rare sexually transmitted disease with an incidence of $0.1 \%$. It is characterized by invasive growth and high recurrence after treatment. Estimated risk of recurrence is 60 to $66 \%$. In some cases malignant transformation is possible and it has been reported in 30 to $56 \%$ of cases [4]. The lesions are triggered by HPV, in particular genotypes 6 and 11 are involved. HPV types 16, 18, 31 and 33 are often found in lesion with neoplastic transformation [6,7]. Occasionally types 16 and 18 are involved too $[6,8]$. Some authors consider poor hygiene and sexual promiscuity some risk factor for the disease. The mean age of patients with giant genital warts is 43 years. The anatomical region most affected by the lesions in the female is the vulva in $\mathbf{9 0 \%}$. Anorectal location is less frequent and sovrapubic localization is rare $[9,10]$. The lesions appear histologically as exophytic mass with epidermal hyperplasia, hyperkeratosis and parakeratosis, without basement membrane disruption but with compression of deep dermis. Immunosuppression can promote the tumour's growth, so every patient with this condition must be checked for HIV, hepatitis virus and syphilis. The patient examined in this work wasn't immunosuppressed Some authors consider giant genital warts (GGW) as precancerous lesions [11] and in any case a correlation between these lesions and verrucous carcinoma is shown, thus making an early radical treatment advisable.

Verrucous carcinoma was first described by Vacker-

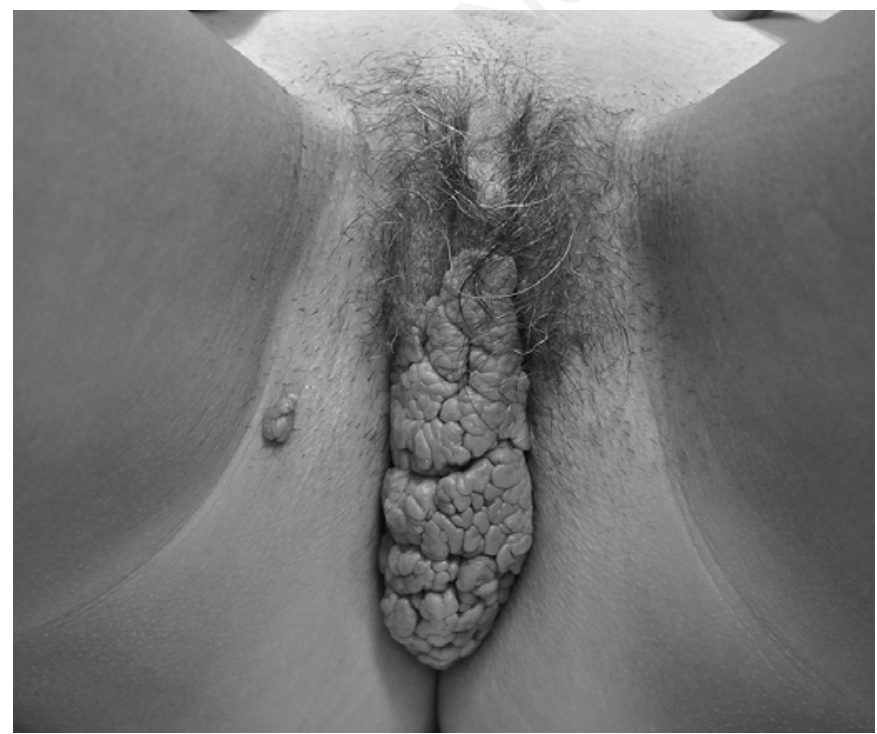

Figure 1. Examination shows cauliflower-like masses diffused on vulvar and perianal region. man in 1948; it is a low grade squamous cell carcinoma with slow and progressive growth, minimal dysplasia and low incidence of metastases. Mortality is estimate about in $20 \%$ of patients [12]. The diagnoses can be difficult due to cytological characteristics [12]. Unlike the Buschke Lowenstein tumour, the verrucous carcinoma shows exophytic and endophytic growth: welldifferentiated squamous cells penetrate the underling tissue across a papillomatous surface. These cellular elements are organized in sinuses and crypts stuffed by keratotic debris [13].

Surgery is one of most relevant option to eradicate

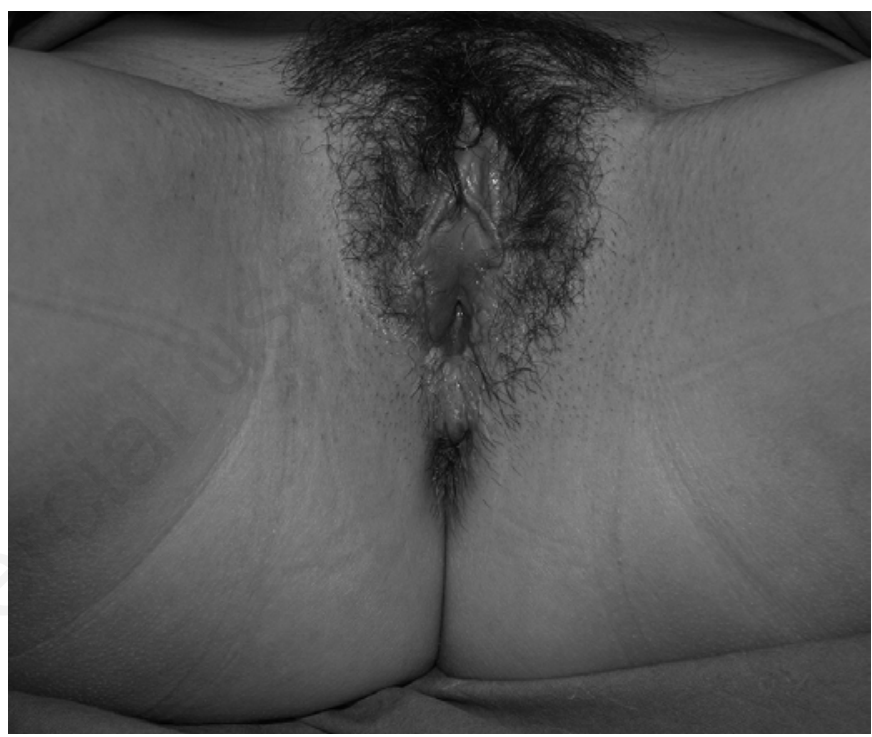

Figure 2. Immediate post-operative photo.

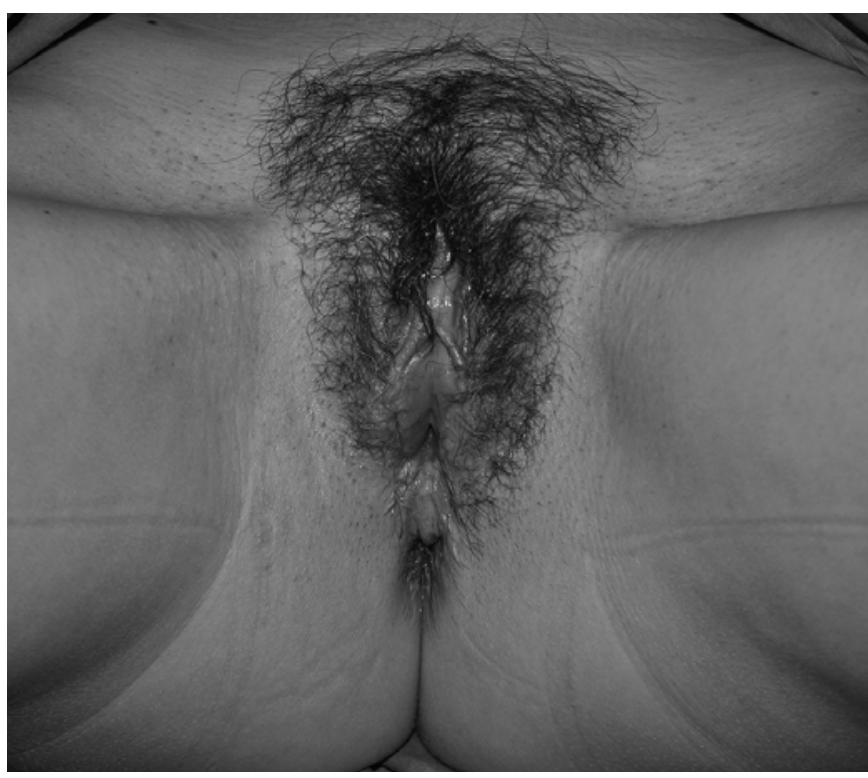

Figure 3. Photo taken after three months. 
a giant genital warts, but particularly for extensive lesions, other therapies such as chemoradiation, topical and intralesional drugs, carbon dioxide laser therapy (etc.) can be used alone or in association to reduce the risk of recurrence [7]. Some authors prefers other treatment such as solution with podofilox solution [14]. Some authors do not prefer harvesting a stoma in these patients, but only prescribing low fibre diet, loperamide and bowel cleansing in post-operative period $[15,16]$.

\section{CONCLUSIONS}

Buschke Lowenstein tumour (BLT) or giant genital warts (GGW) is a sporadic tumour with elevate local recurrence rate. It has a stronger and documented correlation with verrucous carcinoma, so diagnosis and therapy must be accurate and complete. Surgical complete excision remain the main treatment although there are several alternative treatments that, used with surgery, can increase radical rate reducing the risk of recurrence. In some cases, surgery can be very difficult and it must be associated to other strategies. An accurate follow-up is always necessary.

\section{REFERENCES}

1. Buschke A, Lowenstein L. Uber carcinomahliche condylomata accuminata des Penis. Klin Wochenschr 1925;4:1726-8.

2. Mingolla GP, Potì $O$, Carbotta $G$, et al. Reconstructive surgery in anal giant condyloma: report of two cases. Int J Surg Case Rep 2013;4:1088-90.
3. Chu QD, Vereridis MP, Libbey NP, Wanebo HJ. Giant condyloma acuminatum (Buschke Lowenstein tumor) of the anorectal and perianal regions. Dis Colon Rectum 1994;37:950-7.

4. Ahsaini M, Tahiri Y, Tazi MF, et al. Verrucous carcinoma arising in an extended giant condyloma acuminatum (Buschke-Lowenstein tumor): a case report and review of the literature. J Med Case Rep 2013;7:273.

5. Bertram P, Treutner KH, Rubben A, et al. Invasive squamous cell carcinoma in giant anorectal condyloma (Buschke Lowenstein tumor). Langenbecks Arch Chir 1995;380:115-8.

6. Boshart M, zur Hausen H. Human papillomaviruses in BuschkeLöwenstein tumors: physical state of the DNA and identification of a tandem duplication in the noncoding region of a human papillomavirus 6 subtype. J Virol 1986;58:963-6.

7. Lévy A, Lebbe C. Buschke-Löwenstein tumour: diagnosis and treatment. Ann Urol (Paris) 2006;40:175-8.

8. Nthumba PM, Ngure P, Nyoro P. Giant condiloma acuminatum of the scrotum in a man with AIDS: a case report. J Med Case Rep 2011;5:272.

9. El Mejjad A, Amine El M, Mohamed D, et al. Le condylome acuminé géant-tumeur de Buschke Loewenstein (à propos de 3 cas). Prog Urol 2003;13:513-7.

10. Blake PA, Kim CO, Lopez AE, Krongrad A. Verrucous carcinoma of a suprapubic cystostomy track. J Urol 1996;156:174.

11. Gillard P, Vanhooteghem O, Richert B, De La Brasine M. Tumor de Buschke-Loewenstein. Ann Dermatol Venereol 2005;132:98-9.

12. Ciobanu AM, Popa C, Mrcu, Ciobanu CF. Psychotic depression due to giant condyloma Buschke-Lowenstein tumor. Rom J Morphol Embryol 2014;55:189-95.

13. D'Aniello C, Grimaldi L, Meschino N, et al. Verrucous cuniculatum carcinoma of the sacral region. Br J Dermatol 2000;143:445-73..

14. Tyring SK, Arany I, Stanley MA, et al. A randomized, controlled, molecular study of condylomata acuminata clearance during treatment with imiquimod. J Infect Dis 1998;178:551-5.

15. Balik E, Eren T, Bugra D. A surgical approach to anogenital Buschke-Loewenstein tumours (giant condyloma acuminata) Acta Chir Belg 2009;109:612-6.

16. Abbas MA. Wide local excision for Buschke-Löwenstein tumor or circumferential carcinoma in situ. Tech Coloproctol 2011;15:313-8. 